\title{
EL TRATAMIENTO DEL LÉXICO DE ORIGEN GITANO EN EL DICCIONARIO DE AMERICANISMOS DE LA ASOCIACIÓN DE ACADEMIAS DE LA LENGUA ESPAÑOLA
}

\author{
Treatment of the glossary of gypsy origin at the Dictionary of Americanisms \\ of the Association of Spanish Language Academies
}

Ivo Buzek*

\section{RESUMEN}

En el artículo se ofrece un estudio cualitativo del tratamiento de voces de origen gitano en el Diccionario de Americanismos de la Asociación de Academias de la Lengua Española. Se prestará atención a varios aspectos de la microestructura de la obra, sobre todo, en las marcas de uso — sobre todo diatópicas y diafásicas, aunque prestaremos atención también a las indicaciones sobre la vigencia cronológica de los lemas y acepciones en cuestión-y luego contrastaremos el material léxico despojado con los datos que arrojan los corpus académicos, CREA y CORDE, con el objetivo de ver si el tratamiento lexicográfico que reciben las voces en cuestión en el inventario estudiado se corresponde con la documentación textual disponible para ellas y, por consiguiente, si el diccionario — y la Asociación de Academias—ofrecen a los usuarios una imagen y un tratamiento adecuados para esta parcela del léxico español, generalmente olvidado y poco atendido por los lexicógrafos y por los estudiosos en general.

Palabras clave: Gitano-español, americanismos léxicos, lexicografía académica, microestructura, macroestructura, documentación textual.

\begin{abstract}
The aim of the paper is to offer a qualitative study of lexicographic treatment that receive loanwords of Gypsy origin in the Dictionary of Latin American Expressions ("Diccionario de Americanismos") by Association of Academies of the Spanish Language ("Asociación de Academias de la Lengua Española"). Attention will be paid to various aspects of its microstructure, mainly to its usage marcs (geographical area and level of usage, validity and frequency of usage) and the material obtained will be contrasted with the Royal Spanish Academy corpora, CREA and CORDE. The point is to find out if the lexicographic treatment that receives the Gypsy loanwords in the Dictionary corresponds, even only approximately, to their textual documentation available there. Another goal is to prove whether the Dictionary, and the Association of Academies, offers an adequate treatment for this part of Spanish vocabulary to its users. Unfortunately, Gypsy component Spanish lexicon tends to be generally forgotten by lexicographers and scholars in general.
\end{abstract}

Key Words: Spanish Gypsy, Latin American expressions, Academic lexicography, microstructure, macrostructure, textual documentation.

Universidad Masaryk de Brno (Doctorado). Profesor ayudante. República Checa.

Correo electrónico: ibuzek@phil.muni.cz

Recepción: 9/6/2013 Aceptación: 15/11/2013. 


\section{Introducción}

Cada vez que se publica una obra nacida en los talleres de la Real Academia Española o en los de las academias asociadas, las reacciones que se leen y se escuchan después de su presentación van desde unos (auto)elogios incondicionados - y aduladores o hasta serviles-, pasando por unas reflexiones críticas que pretenden ser justas y valorar tanto los aspectos logrados como los no tan logrados de las obras en cuestión, y terminan en unos rabiosos ataques biliosos que niegan ver cualquier aspecto positivo de la publicación, por mínimo que sea.

Como no ha podido ser de otra manera, también el Diccionario de americanismos (DA), promovido por la Asociación de Academias de la Lengua Española y salido en 2010, se ganó en su momento todo tipo de comentarios en los medios de comunicación hispanos en ambos lados del Atlántico. No obstante, nos da la impresión de que esta vez, hasta las fechas que corren ${ }^{1}$, salvo un par de excepciones, se han visto muy pocas reseñas científicas $u$ otras valoraciones críticas y académicamente serias de la obra ${ }^{2}$. En las páginas que siguen por supuesto no pretendemos ofrecer una reseña minuciosa y exhaustiva del diccionario. Nuestros objetivos son mucho más modestos, ya que solamente proponemos prestar atención al tratamiento que recibe en la obra una pequeña parcela del léxico recogido, el del origen gitano o agitanado.

\section{Objetivos y metodología}

Como ya hemos apuntado en el final de la introducción, nuestro objetivo será el estudio del tratamiento lexicográfico que reciben los gitanismos latinoamericanos en el $D A$, pero antes de poner manos a la obra, creemos oportuno aclarar algunos conceptos relacionados con los objetivos y la metodología seguidos en este trabajo.

En primer lugar, advertimos que no es nuestro propósito ofrecer una simple comparación cuantitativa o cualitativa entre los gitanismos documentados en los diccionarios del español europeo - principalmente el Diccionario del español actual (DEA), del equipo de Manuel Seco (1999) - o los correspondientes, en principio, al español general - que serían representados, según manda la tradición, por entradas y acepciones registradas en el DRAE-2001 sin ninguna marcación diatópica que las sitúe en la América Latina-y los documentados en el $D A$, sino que estamos interesados a largo plazo en los gitanismos "latinoamericanos" propiamente dichos, naturalizados en el español en América, y en el tratamiento lexicográfico que estas voces reciben en sus diccionarios ${ }^{3}$.

Somos conscientes de que un diccionario de ismos, como es el $D A$, no será el mejor punto de partida para semejante tarea. Sin embargo, como los proyectos de los diccionarios nacionales del español americano de momento no han dado muchos resultados - salvo el admirable Diccionario del español de México $(D E M)$, del equipo de Luis Fernando Lara (2010), o el Diccionario integral del español de la Argentina (DIEA), coordinado por Federico Plager (2008) —, finalmente hemos optado por acudir también al inventario de las Academias, teniendo en cuenta el prestigio y la autoridad - aunque a veces no exentos de controversiasde los que las academias de la lengua española gozan en el mundo hispánico.

En nuestro estudio nos fijaremos sobre todo en diversos rasgos de microestructura, como son las marcas de uso diatópicas, diafásicas y diastráticas que acompañan las definiciones, igual que en las indicaciones sobre la vigencia cronológica de los lemas y acepciones en cuestión, y luego contrastaremos el material léxico despojado con los datos que arrojan los corpus académicos — CREA y CORDEpara las zonas geográficas indicadas por sus respectivas marcas con el objetivo de ver si el tratamiento lexicográfico que reciben las voces en cuestión en el inventario estudiado se corresponde con la documentación textual disponible para ellas y si el diccionario $-\mathrm{y}$, por consiguiente, la Asociación de Academiasofrece a los usuarios una imagen y un tratamiento adecuados para esta parcela del léxico hispano, 
generalmente olvidado y poco atendido por los lexicógrafos y por los estudiosos en general.

En lo que se refiere al concepto del diccionario, monolingüe en este caso, como género textual y tipo de discurso que es - o debe ser-, estamos plenamente de acuerdo con Lara (2004: 305) en que un diccionario moderno, para poder ejercer su función primaria, que es la de ser un repertorio de consulta fiable y adecuado, en fin, útil para su comunidad de usuarios, debe sustentarse en una sólida base metodológica bien definida $-\mathrm{y}$ explícitamente explicada al público-. En otras palabras, la base de un buen diccionario la debería formar un profundo y serio estudio lexicológico previo. Afirma el investigador mexicano que:

[L]os diccionarios cuentan en su documentación con datos extensos del léxico y, además, precisamente son sus diferentes criterios de selección del vocabulario manifiestos en sus productos, los que exponen con mayor claridad la complejidad del léxico hispánico contemporáneo. En ese sentido, los diccionarios son estudios lexicológicos, que no se limitan a la descripción del vocabulario, sino que lo interpretan dentro de su complejidad social.

Veamos, pues, si en el caso de esta pequeña muestra del léxico que hemos seleccionado el $D A$ cumple con su función de ser un diccionario útil para sus usuarios, demostrando así contar con - o, mejor dicho, ser - un profundo estudio lexicológico, o si, por el contrario, se repite la historia y estamos otra vez ante una recopilación de ismos pintorescos — esta vez más voluminosa, eso sí- cuyas ubicaciones geográficas y niveles de uso asignados $\mathrm{y}$, sobre todo, la vigencia cronológica y la frecuencia de uso atribuidos, están en entredicho.

\section{Los gitanismos en la macroestructura del $D A$}

En primer lugar deberíamos advertir que estamos manejando aquí el término gitanismo en un sentido bastante amplio. Consideramos gitanismo no solamente una palabra de origen inequívocamente romaní, como chavo 'muchacho' o camelear 'engañar', sino que incluimos aquí también las voces con etimologías cruzadas donde una de ellas es gitana, como sería el caso de chingar, en gitano 'pelear', e igualmente las adoptadas por las jergas de la delincuencia, como licar 'observar', que probablemente ya pasarían desapercibidas a la comunidad gitana.

Puesto que no tenemos aquí espacio para entrar en detalle en cuestiones de etimología, remitimos siempre a nuestro estudio monográfico previo sobre el componente gitano en la lengua española y sobre la historia de su documentación en la lexicografía española, sobre todo en la académica (Buzek 2010); datos muy interesantes sobre los gitanismos latinoamericanos, esta vez los mexicanos, se recogen también en Lara 1992a.

En cuanto al número de gitanismos en el español en América, aquí representados por los recogidos en el $D A$, es preciso advertir que no despierta la atención la cantidad del léxico prestado, sino el nivel de su lexicalización y de la adaptación morfofonológica de las voces en cuestión — por no decir "adaptación cultural"$\mathrm{y}$, en el caso de los gitanismos recogidos en el $D A$, las soluciones adoptadas en su tratamiento lexicográfico.

Noqueremos entraraquíen comparaciones, ya hemos dicho más arriba que no pretendemos ofrecer un estudio contrastivo, pero recordemos que el auge de los gitanismos en el español europeo tuvo lugar en la segunda mitad del siglo XIX y en las primeras décadas del siglo XX, es decir, ya en la época de la independencia de las repúblicas hispanoamericanas, cuando el contacto — comercial, cultural y lingüísticoentre la antigua metrópoli y los jóvenes países independientes ya no era tan intenso como antes. También es cierto que el contacto, si lo hubo, fue geográficamente desigual.

De allí se desprende que, si en las fuentes lexicográficas del español europeo encontramos 257 gitanismos en total —incluimos aquí también las voces derivadas-, es lógico que en las fuentes lexicográficas del español en América su número sea menor. Si no estamos del todo equivocados, nuestras búsquedas en 
la nomenclatura del $D A$ han arrojado la cifra de 96 gitanismos. Pero como todos sabemos, las cifras son engañosas y pueden inducir a un error de interpretación. En el caso de los 257 gitanismos en el español de España la gran mayoría son voces poco usadas, si no desusadas ya, e indocumentadas desde hace más de 100 años. Lo problemático allí es que en la lexicografía española - sobre todo en el DRAE-2001-, no van acompañadas con ninguna marca de restricción de uso, poniendo en peligro a los usuarios desprevenidos. Y lo que queremos comprobar es si el mismo tipo de peligro lo corren también los usuarios del $D A$.

La lista de los gitanismos documentados en el $D A$ se ofrece al final del trabajo, en un anexo. En el caso de artículos complejos donde se recogen homónimos bajo un solo lema, indicamos con números romanos, según la práctica ejercida en el diccionario, el ámbito semántico que le corresponde al étimo gitano que se encuentra amalgamado allí.

\section{La microestructura de los gitanismos en el $\boldsymbol{D A}$}

En el siguiente apartado no vamos a estudiar minuciosamente todos los rasgos de la microestructura de los gitanismos documentados en el $D A$, sino que vamos a prestar atención solamente a aquellas características que creemos relevantes para nuestro estudio. En primer lugar nos vamos a dedicar a las marcas diatópicas y vamos a comparar brevemente los resultados con los ofrecidos en Buzek 2009. A continuación hablaremos sobre las marcas sociolingüísticas $\mathrm{y}$ pragmáticas $\mathrm{y}$, finalmente, veremos si la información ofrecida por la microestructura de los artículos en cuestión se corresponde con la documentación textual disponible en los corpus académicos, CREA y CORDE.

Otro rasgo de la microestructura que incluiríamos en nuestro análisis con sumo agrado si pudiéramos, serían los ejemplos. En una de las presentaciones del proyecto, su director, Humberto López Morales (2003: 122), dice que "[e]n todos los lugares donde convenga se añadirán citas o ejemplos". Es de lamentar que ningún caso de los que interesan ahora haya sido considerado conveniente para ponerle ejemplo.

\subsection{Marcas diatópicas}

En cuanto al número de indicaciones geográficas de uso, no es nada sorprendente que las zonas mejor representadas son las que seguían ejerciendo de focos de atracción para la inmigración española incluso después de la independencia, como México, con 62 indicaciones ${ }^{4}$; la zona rioplatense, donde la Argentina obtiene 42 marcas $^{5}$ y Uruguay, $23^{6}$ - muchas veces las dos marcas van juntas-; o Cuba, con 35 marcas 7 .

Estas son las zonas donde los gitanismos parecen gozar de mayor vitalidad, pero es preciso advertir que si se hiciera una comparación entre estos y los gitanismos españoles, cosa en la que no queremos profundizar ahora ${ }^{8}$, se llegaría a verificar que, por ejemplo, en el caso de los gitanismos mexicanos podemos hablar de una serie de derivados "identitarios", típicos del estrato popular y el nivel vulgar de la lengua, como es la familia léxica de chingar, con muy alto índice de documentación, mientras que los demás son términos jergales, probablemente desconocidos fuera de su ámbito social y, en general, difieren bastante tanto formal como semánticamente de los gitanismos españoles.

En el caso de los gitanismos rioplatenses, allí las diferencias formales y semánticas son también notables, se detectan derivados probablemente originados allí y, en comparación con México, difiere también su nivel de uso, ya que parecen ser voces propias de ámbitos coloquiales y están bien documentadas.

Mientras, en Cuba se nota el contacto prolongado con la metrópoli, puesto que los gitanismos cubanos copian muchas veces la forma y el significado de los españoles y la mayoría de ellos no se documenta, según las indicaciones geográficas del $D A$, en otras zonas de Hispanoamérica. 
Si prestamos atención ahora a otras indicaciones geográficas que reciben los gitanismos en el $D A$, notamos que en la América Central su número va disminuyendo, a grandes rasgos, conforme uno se va alejando desde el foco irradiador de la zona, México, y emprende el camino hacia el continente suramericano: para Guatemala hemos encontrado $23 \operatorname{casos}^{9}$, pero en Honduras $35^{10}$, dato que parece contradecir nuestra hipótesis, luego en El Salvador hallamos 27 casos $^{11}$, en Nicaragua $27^{12}$ también, en Costa Rica $5^{13}$ y en Panamá $7^{14}$; luego en Colombia solo $3^{15}$. En casos de gitanismos centroamericanos es bastante habitual que se junten varias marcas geográficas de la zona.

Luego, en la América del Sur la indicación geográfica que parece servir de lazo y de foco de irradiación de gitanismos en el continente es la Argentina. Su influencia se deja sentir, como es obvio, no solamente en Uruguay, sino también en Chile $(22 \text { casos })^{16}$, en Perú (17) ${ }^{17}$ y en Bolivia $(20)^{18}$. Las cifras pueden parecer sorprendentes, dada la orografía de la zona; sin embargo, en el caso de los gitanismos hay que tener en cuenta que es un léxico propio más bien del ámbito urbano, y no rural. El hecho queda confirmado si se compara con Paraguay, zona más bien rural cuyo ambiente urbano es difícilmente comparable con el de Santiago de Chile o el de Lima, y donde registramos solamente 3 gitanismos ${ }^{19}$.

El resto de las zonas geográficas arroja unas cifras moderadas que no sobrepasan 15 ocurrencias y es natural que se junten marcas referentes a países contiguos. En concreto, para Puerto Rico hallamos 11 marcas $^{20}$ - pero para República Dominicana solo dos ${ }^{21}$ _, para Venezuela $15^{22}$ y para Ecuador $8^{23}$; para EE.UU. son finalmente $15^{24}$.

\subsection{Marcas sociolingüísticas y pragmáticas}

Los resultados de búsqueda de marcas sociolingüísticas y pragmáticas tampoco llegan a ser del todo sorprendentes.
La combinación de etiquetas más frecuente -con 67 ocurrencias, si nuestros cálculos son correctos - es la que ubica el uso de la unidad léxica tanto en el nivel sociocultural bajo como en el alto, pero en el segundo se da solo en el estilo espontáneo: $p o p$ + cult $\rightarrow$ espon $^{25}$.

El segundo grupo más numeroso es el de la marca referente al estrato sociocultural popular, pop., con 42 ejemplos ${ }^{26}$; en este caso no se detecta variación del estilo dentro del estrato asignado.

Y el tercer grupo más numeroso de marcas es el que apunta a la valoración social negativa de las voces a las que acompañan, es decir, son voces cuyo uso es considerado vulgar, vulg; son 41 en total ${ }^{27}$. También hemos encontrado un ejemplo de voz con valoración tabú ${ }^{28}$.

Hasta aquí, e insistimos que la comparación es simplemente ilustrativa, el valor diafásico y el de registro de los gitanismos hispanoamericanos coincide a grandes rasgos con los de los gitanismos españoles, tal como lo indican los resultados que aportaron nuestras búsquedas en las columnas del DEA (Buzek, 2010: 239-246).

Antes de pasar a hablar sobre usos menores, creemos necesario advertir que hemos encontrado también 33 voces o acepciones en sus respectivos ámbitos semánticos consideradas neutras, no marcadas $^{29}$, cosa bastante sospechosa, puesto que estamos hablando sobre el léxico prestado de boca de una de las minorías étnicas peor vistas y más menospreciadas en toda la historia de la civilización occidental.

Se podría explicar de dos maneras. En primer lugar, acudiendo a la antigua tesis de que la base lingüística del español de América la formaba el español "vulgar" o "bajo", cuyo léxico estaría marcado en el español europeo pero no en el americano; sin embargo, la tesis trae más problemas que soluciones y no resulta muy convincente como tal. Por tanto, resulta mucho más aceptable aplicar aquí una adaptación de la tesis andalucista, puesto que en las hablas andaluzas el léxico gitano estaba más difundido y menos marcado que en el español de otras áreas, e incluso en el español "general". 
Es cierto que hay casos donde los términos hacen referencia a modalidades de habla, realidades históricas, etc., como en casos de calé o caló. Pero ¿por qué, por ejemplo, en casos de los cubanismos ¡buti!, ¡butín! y butín los primeros dos son estilísticamente neutros, i.e. no marcados, y el tercero está etiquetado como pop + cult $\rightarrow$ espon? Y los corpus académicos no ayudan al usuario a aclarar sus dudas, porque las voces no se recogen allí. Otros ejemplos donde la ausencia de la información sobre el nivel y ámbito de uso puede sorprender bastante al público son, por ejemplo, los de chavalear, chingaderita, chungar o chungón. No hay que ser hispanohablante nativo procedente de las áreas determinadas en cuestión, ni siquiera hace falta ser hispanohablante nativo, para darse cuenta de que no serán, ni mucho menos, unidades léxicas neutras. La razón del tratamiento incoherente se debe a la nómina y a la naturaleza de las fuentes manejadas y también a su característica principal de ser obra contrastiva, como procuraremos explicar en breve.

Pero volvamos ahora a los usos menores. Encontramos aquí, por una parte, etiquetas que apuntan a usos en determinados registros, como delincuencia, delinc (4) ${ }^{30}$; estudiantil, est $(1)^{31}$; y carcelario, $\operatorname{carc}(1)^{32}$; en determinados contextos pragmáticos, como la intención despectiva, desp (2) ${ }^{33}$; o estilos, como el espontáneo, espon $(1)^{34}$.

Por otra parte, hemos localizado también varias combinaciones de marcas, como las referentes a la pertenencia de las voces en cuestión al estrato social popular donde se usan con intención despectiva, pop ${ }^{\wedge} \operatorname{desp}(3)^{35}$; las propias también del estrato popular usadas con intención de hipérbole, pop ${ }^{\wedge}$ hiperb (2) ${ }^{36}$; y una identificada otra vez con estrato popular pero esta vez usada con intención festiva, pop ${ }^{\wedge}$ fest $^{37}$.

Para finalizar el apartado, mencionamos de paso tres marcas referentes a otros ámbitos, al cronológico y al geográfico. Puesto que son muy pocas, hemos decidido incluirlas aquí, de apostilla. El ámbito cronológico está representado por las etiquetas de uso juvenil, juv $(10)^{38}$; obsolescente, obsol (5) ${ }^{39}$; y poco uso, p.u.
$(3)^{40}$. El ambiente geográfico viene reflejado por la marca de uso rural, $\operatorname{rur}(3)^{41}$.

No obstante, los marbetes lexicográficos resultan útiles al usuario solamente cuando reflejan la situación real del uso de las unidades léxicas en cuestión en el momento y en el lugar dados. Y para ello es imprescindible que tanto la macro como la microestructura de la obra cuenten con una sólida base documental textual.

\section{La (in)documentación textual}

Si un usuario hace consultas esporádicas al diccionario, puede que el asunto le pase inadvertido, sin embargo, si las consultas son repetidas y no se limitan a voces procedentes de fuentes literarias, más tarde o temprano le surgirán dudas de diversos tipos: ¿qué frecuencia tienen las voces recogidas? ¿Reflejan las marcas sociolingüísticas y pragmáticas su nivel de uso real? ¿Corresponden las indicaciones geográficas indicadas a sus áreas de uso o, por lo menos, de conocimiento?

Todas estas preguntas tienen una respuesta común: la existencia de una documentación textual que avale tanto la frecuencia, como el nivel y el área geográficos de los usos. Es de lamentar que, acerca de la documentación textual base - los textos auténticos, "reales"ni la "Introducción" ni la "Guía del consultor" de la obra dicen absolutamente nada. Tampoco hay referencia alguna al tema en las presentaciones de la obra que hemos consultado (López Morales 2003 y 2004).

En busca de dicha documentación textual, el usuario acude a los corpus académicos textuales, el CREA y el CORDE, incitado por el hecho de que se menciona el CREA en López Morales 2003 como una fuente base que sirvió de contraste. No obstante, allí le espera una sorpresa bastante desagradable porque de los 96 gitanismos latinoamericanos de nuestro corpus, 52 no figuran en los corpus ${ }^{42}, 31$ de ellos tienen una representación limitada (de 1 a 10 ocurrencias) $)^{43}$ y solo son 13 las voces o ámbitos semánticos —en casos de homónimos - bien representados en los corpus $^{44}$. Hemos buscado 
las voces en cuestión en todos los tipos de textos procedentes de las áreas geográficas indicadas por el $D A$, aunque en repetidas ocasiones hemos extendido el ámbito de búsqueda a las áreas contiguas.

Otras observaciones, pero estas ya serán achacables al método de selección de fuentes para el CREA y el CORDE, es que muchas veces la mayoría de las citas que se obtienen proceden de obras literarias —en repetidas ocasiones incluso de obra u obras de un solo autorque no necesariamente retratarían fielmente el uso "real" cotidiano. También resulta bastante patente que no todas las áreas geográficas del español vienen bien representadas en los corpus. En cuanto a los textos americanos, México y la Argentina, por ejemplo, parecen estar bien representados, pero sospechamos que no estén volcados en los corpus académicos muchos textos cubanos, hondureños o panameños, para mencionar solo algunos.

Mejora la situación si dejamos de manejar los corpus académicos y nos ponemos a buscar las voces en fuentes primarias, a través de navegadores del Internet, en Google Books, etc. De este modo queda confirmado el carácter desequilibrado de los corpus de referencia académicos y, a la vez, queda explicado por qué de momento no han sido aprovechados plenamente sus datos para la confección de nuevos diccionarios académicos.

Pero volvamos a lo que nos interesa ahora, que es la base documental del $D A$. Como ya hemos adelantado más arriba, estamos convencidos de que la documentación textual precaria está condicionada por la naturaleza misma de la base del lemario del $D A$. López Morales apunta en la "Introducción" a la obra que, aparte de los americanismos contenidos en el DRAE-2001 —revisados detenidamente, según afirma el autor-, la nomenclatura "reúne los casi 150 diccionarios de americanismos (generales y nacionales) publicados desde 1975 hasta la fecha, más algunos inéditos aún, todos en formato electrónico con excelentes y ricos índices que facilitan cualquier tipo de búsqueda" (2010: XXVII).
Y allí está el problema: la nomenclatura del $D A$ está apoyada exclusivamente en fuentes lexicográficas - el $D A$ es en realidad un tesoro lexicográfico y no un diccionario de lengua propiamente dicho-, aparentemente carentes de una documentación textual primaria. No hemos encontrado mención alguna de que se haya manejado una base de textos, ni se aclara ningún criterio de frecuencia de aparición que una unidad léxica debe cumplir para poder ser incluida en el lemario del $D A$. Si uno abre otro diccionario dialectal ${ }^{45}$, aunque de otro tipo, el $D E M$, que es integral, se le informa detalladamente sobre los criterios de frecuencias a partir de los cuales una voz llega a figurar en la nomenclatura del diccionario: "Para componer este Diccionario, lo que hemos hecho ha sido tomar todos los vocablos aparecidos en el Corpus [Corpus del español mexicano contemporáneo], cuya frecuencia absoluta fuera al menos de tres apariciones en él" $(D E M, 29)^{46}$.

En cambio, si el usuario del $D A$ no llega a saber dicha información, no obtiene toda la orientación referente al uso de las palabras en cuestión y se queda con las dudas, ignorando si está ante voces normales y corrientes en la región, voces de uso restringido o simplemente ante unas curiosidades locales pintorescas de uso efímero.

\section{Epílogo}

De nuestro paseo por las columnas del $D A$ se puede extraer la siguiente conclusión: el $D A$ no cumple con las exigencias de un usuario seriamente interesado en el léxico hispanoamericano porque el diccionario resulta deficiente ya en su planteamiento básico.

En primer lugar, porque se apoya exclusivamente en documentación lexicográfica de distintas variedades del español en América y no cuenta - probablemente- con ninguna base textual. Y, en segundo lugar, porque toma como referencia la nomenclatura del $D R A E-2001$, de donde fueron vaciadas hacia la base de datos del $D A$ "todas las palabras americanas, tanto las que aparecen con su respectiva marca diatópica 
- un total de 28000 - como las que incluyen una referencia geográfica en el propio texto de la definición, del tipo «árbol muy frecuente en los llanos venezolanos»" (López Morales, 2010: XXVII). En otras palabras, se define el $D A$ como "diferencial ${ }^{47}$ con respecto al español general" ( $D A, \mathrm{XXXI)}$.

Sin embargo, difícilmente se puede confeccionar un diccionario diferencial serio y fiable si su principal referente, el $D R A E$, tiene los defectos que tiene. El DRAE no es un diccionario del español "general" — quedaría por definir qué se entiende bajo tal rótulo-, tampoco es un diccionario del español europeo, no es ni sincrónico ni diacrónico, en fin, es un poco de todo y nada en concreto. Las revisiones de su contenido tampoco parecen ser muy sistemáticas, ya que en la presentación a la vigésima segunda edición del $D R A E$ se le informa al lector que no se tomaron en cuenta los datos del Banco de datos del español y tampoco se llevaron para la edición "algunos preceptos de la Nueva planta" (DRAE-2001, XXXI).

En fin, si sumamos todos los puntos de crítica mencionados hasta el momento, ejemplificados en nuestro pequeño corpus de gitanismos hispanoamericanos, resulta que el concepto teórico del $D A$ como diccionario diferencial y dialectal, tal como se defiende y se presenta en su introducción, así como su valor y utilidad práctica para los usuarios llegan a ser dudosos e insostenibles.

\section{Colofón}

Aunque la imagen ofrecida por el $D A$ ha perdido algo del brillo centelleante que ostentaba en el principio, tampoco hay que verlo todo tan negro. En nuestra opinión, hay dos soluciones posibles al asunto.

La primera está en la línea de los diccionarios diferenciales; no obstante, para las fechas que corren, todos los diccionarios dialectales diferenciales deberían contar con una sólida base textual del área geográfica de su dominio. Si no cuentan con ella, corren el riesgo de ofrecerle al usuario definiciones y orientaciones de uso que, posiblemente, no siempre se corresponderán con la realidad.

También habría que remodelar profundamente la obra de referencia que se manejara para contrastar con ella el material léxico que se pretende recoger en un diccionario diferencial. La primera posibilidad sería actualizar profunda y radicalmente $\mathrm{o}$, mejor dicho, hacer de nueva planta, el diccionario académico. Pero es de suponer que en este caso también habría que reconsiderar el estatus y el papel que deberían jugar las academias de la lengua $y$, sobre todo, su vinculación con la Academia española ${ }^{48}$.

Otra posibilidad, cuyo resultado probablemente sería, al fin y al cabo, bastante similar, sería llevar a cabo un 'diccionario internacional de la lengua española', tal como lo propone Ávila 1998 y 2003, que comprendería sola y exclusivamente el léxico español general, común a todas sus variedades nacionales.

La otra solución posible sería abandonar definitivamente la lexicografía contrastiva y diferencial como tal y apostar decididamente por la integral que comprendería finalmente una serie de diccionarios nacionales, sincrónicos y con sólida base documental para cada uno de ellos. No es ninguna utopía, ya existen representantes que cumplen con estas características: el $D E A$ para el español de España, el $D E M$ para México y DIEA para la Argentina. El proceso sería largo, lento y caro, pero estamos seguros de que los resultados satisfarían (casi) todas las necesidades y dudas de sus usuarios.

Pero todo esto ya es otra historia...

\section{Notas}

1. Mediados del octubre de 2012.

2. Las únicas reseñas o estudios de los que tenemos constancia son los de Cerrón-Palomino 2010, Pelly 2011 y Lara 2012.

3. Después de una tentativa general (Buzek 2009), recientemente hemos ofrecido una primera aproximación a los gitanismos documentados en 
los diccionarios mexicanos contemporáneos en "Presence of Gypsy origin vocabulary in Latin American Spanish varieties: the case of Mexican Spanish and its caló", comunicación leída en 10th International Conference on Romani Linguistics, celebrado en Barcelona de 5 a 7 de septiembre de 2012; una versión extendida del trabajo, en español, saldrá en la revista Études romanes de Brno, vol. 33, $\mathrm{n}^{\mathrm{o}} 2,2012$ (Buzek en prensa).

4. Mx: ¡áchin! I.1; caló I.1.; chaborra I.1.; ¡chale! I.1; chamullar I.1; chava rol; chavalo I.1; chaviza I.1; chavo, -a I.1, chavo, -a II.1, chavo, -a III.1; chingada I.1; a la chingada a.i, a la chingada a.ii; como la chingada b.i; de la chingada c.i, de la chingada c.ii; irse a la chingada a4.i, irse a la chingada a4.ii, irse a la chingada a4.iii, irse a la chingada a4.iv; largarse a la chingada; llevarse la chingada a3.i, llevarse la chingada a3.ii, llevarse la chingada a3.iii; mandar a la chingada; ;me lleva la chingada!; chingadazo I.1, chingadazo I.2; chingadera I.1, chingadera III.1; chingaderita I.1; chingado, -a I.1, chingado, -a I.2; ¡ah, chingado!; chingar I.1, chingar I.2, chingar II.1, chingar II.2, chingar III.1, chingar IV.1, chingar V.1; chinga a tu madre; ¡chínguele!; ¡chíngale!; chingazo I.1; al chingazo; al puro chingazo; chingón, na I.1, chingón, na II.2; chingonería I.1; licar I.1; mangonear I.1; mangoneo I.1; sandunga I.1, sandunga I.2; sandunguero, -a I.1; zandunga I.1, zandunga I.2; zandunguear I.1; zandunguero, -a I.1.

5. Ar: camelear I.1; camelero I.1; chamullador, -ra I.1; chamullar I.2; chamullero, -a I.2, chamullero, -a I.3; chamuyada I.1; chamuyador, -ra I.1; chamuyar I.1, chamuyar II.1; chamuyero, -a I.1, chamuyero, -a I.2; chamuyo I.1, chamuyo I.2, chamuyo II.1; chingada II.1; chingar VI.1, chingar VI.2; chingarla; chingarle; chingue I.1, chingue I.2, chingue I.3; chorear(se) I.1; choreo I.1; choro, -a I.1; chorro, -a I.1; currar I.1, currar I.2, currar II.1; curro I.1, curro II.1; junar I.1, junar I.2; manganeta I.1; mangar I.1; manguear III.1; manguero, -a II.1; pirarse I.1, pirarse I.2; pire I.1; sandunguear I.1.

6. Ur: camelero I.1; chamuyada I.1; chamuyador, ra I.2; chamuyar I.1, chamuyar II.1; chamuyo I.1, chamuyo I.2; chingar VI.1, chingar VI.2; chorear(se) I.1; choreo I.1; chorro, -a I.1; curro I.1; junar I.1, junar I.2; manganeta I.1; mangar I.1; manguear III.1, manguear III.2; manuero, -a II.1; pirarse I.1, pirarse I.2; pire I.1.

7. Cuba: ¡buti! I.1; butín I.1; ¡butín! I.1; chamullar I.3; choro, -a I.2; curralar I.1; curralo I.1; fula I.1; fulastre I.1; fulastrería I.1; fulastrón I.1; gao

I.1; giña I.1; jama I.1; jamar(se) I.1, jamar(se) I.2, jamar(se) II.1, jamar(se) III.1, jamar(se) IV.1; jamar con papa; jiña I.1, jiña II.1; tener jiña; mangada I.1; mangadera I.1; mangar II.1; mangonear I.1; mangoneo I.1; parguela I.1; pura I.1; pureta I.1; pureto I.1; puro III.1; sandunguear I.1; sandungueo I.1.

8

10. Ho: caló I.1; chavalo I.1; chaviza I.1; chavo, -a I.1, chavo, -a II.1; chingada III.1; a la chingada a.i; en la chingada grande d; irse a la chingada grande b4; largarse a la chingada; chingadera I.2, chingadera I.3; chingado, -a I.3, chingado, -a I.4; chingador, ra I.1, chingador, ra II.1; chingar III.1, chingar VII.1; chingón, na I.1, chingón, na I.2, chingón, na I.3, chingón, na I.4, chingón, na I.5, chingón, na I.6, chingón, na II.1; chinguearse I.1, chinguearse I.2, chinguearse I.3; jaño, -a I.1, jaño, -a I.2; sandunguear I.2; sandungueo I.1, sandungueo I.2; sandunguero, -a I.4; zandunguero, -a I.2.

11. ES: ¡chale! II.1; chaviza I.1; a la chingada a.i; de la chingada c.iii; irse a la chingada grande b4; largarse a la chingada; ¡me lleva la chingada!; chingadera I.2, chingadera I.3, chingadera IV.1; chingado, -a I.1, chingado, -a I.2; chingador, ra I.1, chingador, ra II.1; chingar I.1, chingar I.2, chingar III.1, chingar VII.1; chingón, na I.1, chingón, na I.2, chingón, na I.3, chingón, na II.1; chungar I.1; chungón, na I.1; chunguear I.1; jama I.1; jaña I.1.

12. Ni: caló I.1; chavalear I.1; chavalo I.1; chavo, -a I.1, chavo, -a II.1; a la chingada a.i; en la chingada grande d; irse a la chingada a4.i, irse a la chingada a4.v; irse a la chingada grande b4; largarse a la chingada; mandar a la chingada; chingadera I.2; chingado, -a I.3, chingado, -a I.4; chinguearse I.1; jama I.1; jaña I.1; jaño, -a I.1, jaño, -a I.2; jiña III.1; mangonear I.1; mangoneo I.2; sandunguear I.2; sandungueo I.1, sandungueo I.2.

13. CR: chavalo I.1; chingadera I.2, chingadera II.1; chingar VIII.1; jama I.1. 
14. Pa: de la chingada c.iii; chingar VIII.1; chingazo I.2; chingón, na I.4; mangar II.1; mangonear I.1, mangonear II.1.

15. Co: chaborro, -a I.1; chorear(se) I.1; mangonear I.2.

16. Ch: chamullador, ra I.1; chamullento, -a I.1, chamullento, -a I.2; chamullero, -a I.1; chamuyo I.1, chamuyo II.1; chingar I.1, chingar I.2; choreada II.1; choreador, ra I.1; chorear(se) I.1; choreo I.1; choro, -a I.1; sandunga II.1; sandunguear I.1; sandungueo I.1, sandungueo I.2, sandungueo II.1; sandunguero, -a I.2, sandunguero, -a I.3; zandunga II.1; zandunguear I.1.

17. Pe: chamullador, ra I.1; chamullero, -a I.1; chamuyador, ra I.1; chamuyar I.1; chamuyero, -a I.1; chamuyo I.1; choreador, ra I.1; chorear(se) I.1; choreo I.1; choro, -a I.1; jama I.1; jamear I.1; mangoneo I.2; sandunguear I.1; sandungueo II.1; sandunguero, -a I.2, sandunguero, -a I.3.

18. Bo: chamuyador, ra I.1; chamuyar I.1, chamuyar I.2; chamuyo I.1, chamuyo I.2; chorro, -a I.2; chingarla; choreada II.1; choreador, ra I.1; chorear(se) I.1; choreo I.1; choro, -a I.1; manguear I.1; mangar I.1; mangonear I.1; manguear III.1, manguear III.2; manguero, -a II.1; sandunguero, -a I.2; zandunga III.1.

19. Py: chingar VI.1; chorro, -a I.1; manguear III.1.

20. PR: chanarse I.1, chanarse II.1; chingón, na III.1; chunguero, -a I.1; mangoneo I.1; sandunga II.1; sandunguear I.1; sandungueo I.1, sandungueo I.2, sandungueo II.1; sandunguero, -a I.2, sandunguero, -a I.4.

21. RD: chungo II.1; mangoneo I.1.

22. Ve: chaborro, -a I.1; chavalo I.1; chorea(se) I.1; choreo I.1; choro, -a I.1; jama I.1; pargolete I.1; pire I.2; pure I.1, pure I.2, pure I.3; pureta I.1; pureto I.1; pureto, -a I.1; zandunguero, -a I.1.

23. Ec: calé I.1; chorear(se) I.1; choreo I.1; choro, -a I.1; jama I.1; jamear I.1; mangonear I.1; mangoneo I.1.

24. EE.UU.: a la chingada a.i, a la chingada a.ii; de la chingada c.i, de la chingada c.ii; chingado, -a I.1; chingado, -a I.2; chingar I.1, chingar I.2, chingar II.1, chingar II.2, chingar III.1, chingar IV.1; chinga a tu madre; ¡chíngales!; parguela I.1.
25. pop + cult $\rightarrow$ espon: ¡áchin! I.1; ¡butín! I.1; chaborro, -a I.1; chamullador, ra I.1; chamullar I.2; chamullento, -a I.2; chamullero, -a I.1, chamullero, -a I.2, chamullero, -a I.3; chamullo I.1, chamullo II.1, chamullo II.2, chamullo II.3; chamuyar I.1, chamuyar II.1; chamuyo I.1, chamuyo I.2, chamuyo II.1; chanarse II.1; chavalo I.1; chaviza I.1; chingada III.1; chingadera I.2, chingadera II.1; chingar I.1, chingar I.2; chingón, na I.2, chingón, na III.1; choreada II.1; choreador, ra I.1; chorear(se) I.1; choreo I.1; chorro, -a I.1; chunguero, -a I.1; curralar I.1; curralo I.1; currar I.1, currar I.2, currar II.1; curro I.1, curro II.1; jama I.1; junar I.1, junar I.2; licar I.1; manganeta I.1; mangonear I.1; mangoneo I.1; manguear III.2; manguero, -a II.1; pirarse I.1, pirarse I.2; pura I.1; pureto, -a I.1; puro III.1; sandunga II.1; sandunguear I.1; sandungueo I.1, sandungueo I.2, sandungueo II.1; sandunguero, -a I.2, sandunguero, -a I.3; zandunga II.1; zandunguear I.1; zandungueo I.1.

26. pop: camelear I.1; camelero I.1; chamuyada I.1; chamuyador, ra I.1; chava rol; chingada II.1; a la chingada a.i; como la chingada b.i; chingar la pita; chingarla; chingazo I.1, chingazo I.2; chingón, na I.1, chingón, na I.3, chingón, na I.4, chingón, na I.5, chingón, na I.6; chingonería I.3; chingue I.1, chingue I.2, chingue I.3; chinguearse I.2, chinguearse I.3; chorear(se) I.1; choro, -a I.1; fulastrería I.1; gao I.1; jamar(se) I.1, jamar(se) I.2, jamar(se) III.1, jamar(se) IV.1; jamear I.1; jiña I.1; tener jiña; mangar I.1; mangonear I.2; manguear III.1; parguela I.1; sandungueo I.1; sandunguero, -a I.2; zandunga III.1; zandunguear I.1.

27. vulg: chanarse II.1; chingada I.1; a la chingada a.ii; como la chingada b.ii; de la chingada c.i, de la chingada c.ii, de la chingada c.iii; en la chingada grande d; irse a la chingada a4.i, irse a la chingada a4.ii, irse a la chingada a4.iii, irse a la chingada a4.iv, irse a la chingada a4.v; llevar la chingada a3.i, llevar la chingada a3.ii, llevar la chingada a3.iii; mandar a la chingada; ¡me lleva la chingada!; chingadazo I.1, chingadazo I.2; chingadera I.3, chingadera III.1, chingadera IV.1; chingado, -a I.1, chingado, -a I.2, chingado, -a I.3, chingado, -a I.4; jah, chingado!; chingador, ra II.1; chingar I.1, chingar I.2, chingar II.1, chingar II.2, chingar III.1, chingar IV.1, chingar V.1; chinga a tu madre; choreada II.1; choreador, ra I.1; chorear(se) I.1; jiña I.1.

28. tabú: chingón, na III.1.

29. Ø: irse a la chingada grande b4; largarse a la chingada; chingadera I.1; chingaderita I.1; chingar VI.1, chingar VI.2; ¡chínguele!; chingarle; ¡chíngale!; 
¡chíngales!; chinguear(se) I.1; chungar I.1; chungón, na I.1; chunguear I.1; giña I.1; jamar(se) II.1; jiña II.1; manganear I.1; manganía I.1; mangoneo I.2; pire I.2; pureta I.1; pureto I.1; sandunga I.1, sandunga I.2; sandunguear I.2; sandunguero, -a I.1, sandunguero, -a I.4; zandunga I.1, zandunga I.2; zandunguero, -a I.1, zandunguero, -a I.2.

30. delinc: ;chale! II.1; chorear(se) I.1; choro, -a I.2; pargolete I.1.

31. est: chingar VII.1.

32. carc: jaña I.1.

33. desp: chungo II.1; fula I.1.

34. espon: mangar I.1.

35. pop ${ }^{\wedge}$ desp: chingón, na II.1; fulastre I.1; fulastrón I.1.

36. $\operatorname{pop}^{\wedge}$ hiperb: al chingazo; al puro chingazo.

37. $\operatorname{pop}^{\wedge}$ fest: jamar con papa.

38. juv: chanarse I.1; chanarse II.1; ;chíngales!; jama I.1; jaño, -a I.1; jaño, -a I.2; pire I.1; pure I.1, pure I.2, pure I.3.

39. obsol: calé I.1; chaborra I.1; mangada I.1; mangadera I.1; mangar II.1.

p.u: chamuyador, ra I.1; chorear(se) I.1; choreo I.1.

41. rur: chaborra I.1; chingador, ra I.1; chingar VIII.1.

42. Sin documentación: ¡áchin!; ¡buti!; butín; ¡butín!; camelear; camelero, -a; chaborra; chaborro, -a; chamullador, -ra; chamullar; chamullero; chamuyada; chamuyador; chamuyero, -a; chanarse; chava rol; chavalear; chingador, -ra; chingue I.; chinguearse I.; choreada II.; choreador, -ra; choreo I.; chungar; chungo II.; chungón, -na; chunguear I.; chunguero, -a; curralar; curralo; currar; fula; fulastre; fulastrería; fulastrón; gao; giña; jamar(se); jamear; jaña I.; jaño, -a; jiña; mangada; mangadera; manganear; manganía; mangar I., II.; pargolete; parguela; pire; pure; pureto.

43. 1-10 casos: calé; caló; ¡chale!; chamullento; chamullo; chamuyar; chamuyo; chaviza; chingaderita; chingazo; chingonería; chorear I.; choro, -a I.; curro I., II.; jama; junar; licar; mangonear I.; mangoneo I.; manguear III.; manguero, -a II.; pirarse; pureta; pureto, -a; sandunga; sandunguear; sandungueo; zandunga; zandunguear; zandungueo; zandunguero, -a.

44. Más de 10 casos: chavalo; chavo, -a; chingada; chingadazo; chingadera; chingado,-a; chingar(se); chingón, -na; chorro, -a I.; sandunguero, -a.

45. En el sentido estricto de la palabra, el DEM no se debería denominar "dialectal" porque, de hacerlo, se desvirtúa de su finalidad. Es más conveniente considerarlo nacional, una especie de "Webster mexicano", como dice Lara, con un poco de exageración, en la "Introducción" a la obra (2010: 15). Para las necesidades del trabajo, nos servimos de la tipología de diccionarios presentada en Campos Souto y Pérez Pascual 2003

46. Pero es cierto que el criterio de frecuencia de aparición de las voces en el Corpus referido iba sufriendo adaptaciones. En una de las presentaciones del proyecto del DEM (Lara 1992b), su director decía que "[p]ara el DEM definitivo tomaremos en cuenta todos los vocablos cuya frecuencia absoluta sea mayor o igual a dos".

47. La letra cursiva es de la fuente citada.

48. Una serie de análisis muy valientes, algunos brillantes, y también varias propuestas de mejora, se ofrecen en el volumen colectivo El dardo en la Academia (Senz y Alberde 2011).

\section{Bibliografía}

Asociación de Academias de la Lengua Española. (2010). Diccionario de americanismos. Madrid: Santillana.

Ávila, Raúl. (1998). “Españolismos y mexicanismos: hacia un diccionario internacional de la lengua española". En: Nueva Revista de Filología Hispánica XLVI (2): 395-406.

Ávila, Raúl. (2003). "Diccionarios locales, nacionales, internacionales". En: 
Revista Internacional de Lingüística Iberoamericana I: 51-66.

Buzek, Ivo. (2009). "Léxico gitano documentado en las variedades latinoamericanas del español". En: Études romanes de Brno XXX (2): 187-202.

Buzek, Ivo. (2010). La imagen del gitano en la lexicografía española. Brno: Masarykova univerzita.

Buzek, Ivo. (2012). Presence of Gypsy origin vocabulary in Latin American Spanish varieties: the case of Mexican Spanish and its caló. $10^{\text {th }}$ International Conference on Romani Linguistics. Barcelona, September 5-7 2012. http://muni. academia.edu/IvoBuzek/Papers/1935215/ Presence_of_Gypsy_origin_vocabulary_ in_Latin_American_Spanish_varieties the case of Mexican Spanish and its calo. Consulta: 2012-10-19.

Buzek, Ivo. (En prensa). Presencia del léxico de origen gitano en las variedades latinoamericanas del español: el caso del español de México y su caló". En: Études romanes de Brno XXXIII (2).

Campos Souto, Mar y José Ignacio Pérez Pascual. (2003). "El diccionario y otros productos lexicográficos". En: Medina Guerra, Antonia María (coord.). Lexicografía española. Barcelona: Ariel: 53-78.

Cerrón-Palomino, Rodolfo. (2010). “Acotaciones al Diccionario de americanismos". En: Lexis XXXIV (1): 161-176.

Lara, Luis Fernando. (1992a). "El caló revisitado". En: Luna Traill, Elizabeth (ed.). Scripta Philologica in Honorem Juan M. Lope Blanch a los 40 años de docencia en la UNAM y a los 65 años de vida. Tomo 2. Lingüistica española e iberoamericana. México: Universidad
Nacional Autónoma de México, Instituto de Investigaciones Filológicas: 567-592.

Lara, Luis Fernando. (1992b). "Teoría y método del Diccionario del español de México". En: Actas del congreso de la lengua española (Sevilla, 1992). Madrid: Instituto Cervantes: 660-665. http://cvc. cervantes.es/obref/congresos/sevilla/ unidad/ponenc_lara.htm. Consulta: 201210-19.

Lara, Luis Fernando. (2004). "Culturas nacionales y léxico contemporáneo del español". En: Lüdtke, Jens y Christian Schmitt (eds.). Historia del léxico español. Enfoques y aplicaciones. Homenaje a Bodo Müller. Madrid/Frankfurt am Main: Iberoamericana/Vervuert: 303-315.

Lara, Luis Fernando (dir.). (2010). Diccionario del español de México. 2 tomos. México: El Colegio de México, Centro de Estudios Lingüísticos y Literarios.

Lara, Luis Fernando. (2012). "Diccionario de americanismos". En: Panace@. Revista de Medicina, Lenguaje y Traducción XIII (36): 352-255. http://www.tremedica.org/ panacea/IndiceGeneral/n36-plumero_ LFLara.pdf. Consulta: 2013-01-12.

López Morales, Humberto. (2003). “Diccionario académico de americanismos. Síntesis de planta”. En: Martín Zorraquino, María Antonia y José Luis Aliaga Jiménez (eds.). La lexicografía hispánica ante el siglo XXI. Balance y perspectivas (Actas del Encuentro de Lexicógrafos celebrado en Zaragoza, en el marco del Centenario María Moliner, los días 4 y 5 de noviembre de 2002). Zaragoza: Departamento de Educación, Cultura y Deporte / Institución Fernando el Católico: 115-127. 
López Morales, Humberto. (2004). "El

Diccionario académico de americanismos: tradición e innovación". En Ahumada, Ignacio (ed.). Lexicografía regional del español. VI Seminario de Lexicografía Hispánica, Jaén, 19 al 21 de noviembre de 2003. Jaén: Universidad de Jaén: 19-36.

López Morales, Humberto. (2010). "Introducción". En: Asociación de Academias de la Lengua Española. Diccionario de americanismos. Madrid: Santillana: XXVII-XXVIII.

Pelly, María Elena. (2011). "Asociación de Academias de la Lengua Española. Diccionario de americanismos. Lima: Santillana Ediciones Generales, 2010. Pp. 2333. ISBN 978-84-294-9550-8". En: Hispania XCIV (3): 548-549.

Plager, Federico (coord.). (2008). Diccionario integral del español de la Argentina. Buenos Aires: Voz activa. http://www. voz-activa.com.ar/flipdiccionario/. Consulta: 2012-10-19.

Real Academia Española (2001): Diccionario de la Lengua Española, $22^{a}$ ed., Madrid, Espasa-Calpe.

Real Academia Española. Banco de datos (CREA) [en línea]. Corpus de referencia del español actual. http://www.rae.es.

Real Academia Española. Banco de datos (CORDE) [en línea]. Corpus diacrónico del español. http://www.rae.es.

Seco, Manuel, Gabino Ramos y Olimpia Andrés. (1999). Diccionario del español actual. 2 tomos. Madrid: Aguilar.

Senz, Silvia y Montserrat Alberde (eds.). (2011). El dardo en la Academia. 2 tomos. Barcelona: Melusina.

\section{Anexo}

¡áchin!

¡buti!

butín

¡butín!

calé

caló

camelear

camelero, -a

chaborra

chaborro, -a

¡chale!

chamullador, -ra

chamullar

chamullento

chamullero, -a

chamullo

chamuyada

chamuyador, -ra

chamuyar

chamuyero, -a

chamuyo 


\begin{tabular}{|c|c|}
\hline chanarse & choro, -a I. \\
\hline chava rol & chorro, -a I. \\
\hline chavalear & chungar \\
\hline chavalo & chungo II. \\
\hline chaviza & chungón, -na \\
\hline chavo, -a & chunguear I. \\
\hline chingada & chunguero, -a \\
\hline chingadazo & curralar \\
\hline chingadera & curralo \\
\hline chingaderita & currar \\
\hline chingado,-a & curro I., II. \\
\hline chingador, -ra & fula \\
\hline chingar(se) & fulastre \\
\hline chingazo & fulastrería \\
\hline chingón, -na & fulastrón \\
\hline chingonería & gao \\
\hline chingue I. & giña \\
\hline chinguearse I. & jama \\
\hline choreada II. & jamar(se) \\
\hline choreador, -ra & jamear \\
\hline chorear(se) I. & jaña I. \\
\hline choreo I. & jaño, -a \\
\hline
\end{tabular}


jiña

junar

licar

mangada

mangadera

manganear

manganeta

manganía mangar I., II.

mangonear I.

mangoneo I.

manguear III.

manguero, -a II

pargolete

parguela

\section{(c) $($ ) $९$}

Este obra está bajo una licencia de Creative Commons

Reconocimiento-NoComercial-SinObraDerivada 4.0 Internacional. 
